\title{
Anti-Angiogenesis as a New Concept for the Therapy of Neovascular Diseases
}

\author{
L. Schweigerer \\ Sektion Onkologie/Immunologie, Universitäts-Kinderklinik, Heidelberg
}

During angiogenesis - the sprouting of new capillaries from existing blood vessels - endothelial cells of an existing vessel invade the surrounding tissues and proliferate at the tip of the new capillary. Both processes are continuously repeated until a new blood capillary is generated.

In the healthy organism, angiogenesis is self-limited and restricted to a few conditions such as corpus luteum development or placental growth. Otherwise angiogenesis is virtually non-existent: of all vascular endothelial cells present in the healthy organism, only 0.1 percent or fewer actively proliferate. All other endothelial cells are in a resting state, and their turnover rate is measured in months to years [2].

However, in pathologically altered tissues, angiogenesis is dramatically enhanced and no longer self-limited: ten percent or more of the vascular endothelial cells in these tissues do actively proliferate [2]. This opens up the opportunity for selective therapeutic inhibition of capillaries proliferating in the diseased tissues [7]. Pathological angiogenesis is seen during the development and progression of various diseases, including rheumatoid arthritis, psoriasis, bartonellosis, the rejection of organ transplants, retrolental fibroplasia, diabetic retinopathy, hemangiomas, and many more diseases. Probably one of the most impressive manifestations of angiogenesis is seen in the vicinity of solid tumors; it is then called tumor angiogenesis.

So far, much of the pathological basis for tumor angiogenesis is unknown. However, through the pioneering work of Judah Folkman it has become clear that tumor angiogenesis can be mediated by soluble molecules called angiogenesis factors [3]. Thus far about a dozen or more such factors have been identified, the most prominent and best characterized being basic fibroblast growth factor (bFGF) [8], one of the eight currently known members of the fibroblast growth factor family [5]. Some of the bFGF-related angiogenic molecules are products of oncogenes, including INT2, HST/ KFGF, FGF5, and FGF6. These molecules are present in many normal tissues where their activity appears to be tightly controlled. When these controls break down, however, the oncogenes may unleash runaway activity, eventually resulting in tumor angiogenesis.

Until recently it was thought that inadequate activity of such oncogene-encoded angiogenic molecules might play the main role in tumor angiogenesis. However, recent data suggest that angiogenesis is the result of a delicate balance between growth-stimulatory and -inhibitory molecules, represented by the angiogenesis factors [3] and angiogenesis inhibitors [1]. Although little known, the therapeutic effects of some established cytostatic agents, such as methothrexate, daunorubicin, and adriamycin, appear to be mediated, at least in part, by the inhibition of tumor angiogenesis. However, the serious side effects of these agents are disadvantageous. This may not be the case with endogenous anti-angiogenic compounds. Some of these compounds - vitamin D3 and retinoic acid, for example - are well known, but their anti-angiogenic properties have only recently been recognized. Other recently discovered endogenous anti-angiogenic compounds include thrombospondin, platelet factor-4, cartilage-derived inhibitor, tumor necrosis factor- $\alpha$ [1], and tetrahydrocortisol [3]. Tetrahydrocortisol is present in human blood and urine, from which other extremely potent endogenous anti-angiogenic substances have also recently 
been isolated (T. Fotsis and L. Schweigerer, unpublished). Interestingly, some of these substances are of dietary origin, suggesting a possible explanation for the long-proposed relationship between diet and tumor development.

A recent international symposium in St. Gallen (Switzerland) on angiogenesis and its therapeutic modulation ("Angiogenesis: Key Principles, Science, Technology and Medicine") has revealed more insight into ways to prevent pathological angiogenesis. There are increasing attempts to identify and develop substances aimed at fighting neovascular diseases. One of the more promising candidates is fumagillin, a substance derived from a fungus discovered as a growth-inhibitory contaminant of vascular endothelial cell cultures [4]. In the meantime, more potent analogs have been developed which inhibit angiogenesis in vivo at extremely low concentrations, while at the same time, but with higher concentrations, also inhibiting tumor growth $[4,6]$. These analogs are currently being analyzed in phase 1 and 2 studies, and clinical trials will soon follow (J. Folkman, personal communication).

As more and more anti-angiogenic compounds are found, anti-angiogenesis will soon become established as a new concept for the therapy of angiogenic diseases. There are many indications for anti-angiogenic therapy in virtually all fields of medicine: diabetic retinopathy in internal medicine, abdominal adhesions in surgery, rejection of cornea transplants in ophthalmology, angiofibro- ma in otolaryngology, hemangioma in pediatrics, and solid tumors in all clinical specialties.

\section{References}

1. Bouck NP (1990) Tumor angiogenesis: the role of oncogenes and tumor suppressor genes. Cancer Cells 2:179-185

2. Denekamp J (1990) Vascular attack as a therapeutic strategy for cancer. Cancer Met Rev 9:267-282

3. Folkman J, Klagsbrun M (1987) Angiogenic factors. Science 235:442-447

4. Ingber D, Fujita T, Kishimoto S, Sudo K, Kanamaru T, Brem H, Folkman J (1990) Synthetic analogues of fumagillin that inhibit angiogenesis and suppress tumour growth. Nature 348:555-557

5. Klagsbrun M (1990) The fibroblast growth factor family: structural and biological properties. Prog Growth Factor Res $1: 207-235$

6. Kusaka M, Sudo K, Fujita T, Marui S, Itoh F, Ingber D, Folkman J (1991) Potent anti-angiogenic action of AGM1470: comparison to the fumagillin parent. Biochem Biophys Res Commun 174:1070-1076

7. Schweigerer L (1990) Anti-Angiogenese als neues Konzept zur Therapie ungehemmter Neovaskularisation. Dtsch Aerztebl $50 \mathrm{~A}: 4050$

8. Schweigerer L (1990) Basic fibroblast growth factor: properties and clinical implications. In: Habenicht AJR (ed) Growth factors, differentiation factors, and cytokines. Springer, Berlin Heidelberg New York, pp 42-55

Dr. Lothar Schweigerer

Sektion Onkologie/Immunologie

Universitäts-Kinderklinik

Ruprecht-Karls-Universität

Im Neuenheimer Feld 150

W-6900 Heidelberg, FRG 